\title{
Ramosetron versus Palonosetron in Combination with Aprepitant and Dexamethasone for the Control of Highly-Emetogenic Chemotherapy-Induced Nausea and Vomiting
}

\author{
Jin Hyoung Kang, MD, $P h D^{1}$ \\ Jung Hye Kwon, MD, PhD² \\ Yun-Gyoo Lee, MD, PhD ${ }^{3}$ \\ Keon Uk Park, MD, PhD ${ }^{4}$ \\ Ho Jung An, MD, PhD \\ Joohyuk Sohn, MD, PhD ${ }^{6}$ \\ Young Mi Seol, MD, PhD \\ Hyunwoo Lee, MD \\ Hwan-Jung Yun, MD ${ }^{9}$ \\ Jin Seok Ahn, MD, PhD'10 \\ Ji Hyun Yang, MD ${ }^{1}$ \\ Hunho Song, MD, PhD² \\ Dong-Hoe Koo, MD, PhD ${ }^{3}$ \\ Jin Young Kim, MD, $\mathrm{PhD}^{4}$ \\ Gun Min Kim, MD, PhD \\ Hwa Jung Kim, MD, PhD ${ }^{11}$ \\ *A list of author's affiliations appears at the \\ end of the paper. \\ Correspondence: Jung Hye Kwon, MD, PhD \\ Division of Hemato-Oncology, Department of \\ Internal Medicine, Kangdong Sacred Heart \\ Hospital, Hallym University College of \\ Medicine, 150 Seongan-ro, Gangdong-gu, \\ Seoul 05355, Korea \\ Tel: 82-2-2225-2788 \\ Fax: 82-2-477-6925 \\ E-mail: kwonjhye@naver.com \\ Received November 20, 2019 \\ Accepted March 17, 2020 \\ Published Online March 18, 2020
}

\begin{abstract}
Purpose
The purpose of this study was to compare ramosetron (RAM), aprepitant (APR), and dexamethasone (DEX) [RAD] with palonosetron (PAL), APR, and DEX [PAD] in controlling highlyemetogenic chemotherapy (HEC)-induced nausea and vomiting.
\end{abstract}

\begin{abstract}
Materials and Methods
Patients were randomly assigned (1:1) to receive RAD or PAD:RAM (0.3 mg intravenously) or PAL (0.25 mg intravenously) D1, combined with APR (125 mg orally, D1 and 80 mg orally, D2-3) and DEX (12 mg orally or intravenously, D1 and 8 mg orally, D2-4). Patients were stratified by sex, cisplatin-based chemotherapy, and administration schedule. The primary endpoint was overall complete response (CR), defined as no emesis and no rescue regimen during 5 days of HEC. Secondary endpoints were overall complete protection (CP; CR+nausea score $<25 \mathrm{~mm}$ ) and total control (TC; CR+nausea score $<5 \mathrm{~mm}$ ). Quality of life was assessed by Functional Living Index Emesis (FLIE) questionnaire on D0 and D6.
\end{abstract}

\section{Results}

A total of 279 patients receiving RAD $(n=137)$ or PAD $(n=142)$ were evaluated. Overall CR rates in RAD and PAD recipients were $81.8 \%$ and $79.6 \%$ (risk difference [RD], 2.2\%; 95\% confidence interval [Cl], -7.1 to 11.4$)$, respectively. Overall $\mathrm{CP}$ and TC rates for RAD and PAD were $56.2 \%$ and $58.5 \%$ (RD, $-2.3 \%$; $95 \% \mathrm{Cl},-13.9$ to 9.4 ) and $47.5 \%$ vs. $43.7 \%$ (RD, $3.8 \%$; $95 \% \mathrm{Cl},-7.9$ to 15.5 ), respectively. FLIE total score $\geq 108$ (no impact on daily life) was comparable between RAD and PAD (73.9\% vs. 73.4\%, respectively). Adverse events were similar between the two groups.

\section{Conclusion}

In all aspects of efficacy, safety and quality of life, RAD is non-inferior to PAD for the control of chemotherapy-induced nausea and vomiting in cancer patients receiving HEC.

\section{Key words}

Ramosetron, Palonosetron, Aprepitant, Antiemetics, Nausea, Vomiting, Neoplasms

\section{Introduction}

Chemotherapy-induced nausea and vomiting (CINV) has been reported as the fifth most feared symptom in cancer patients receiving systemic cytotoxic chemotherapy [1,2]. Overwhelming evidence shows that CINV, when not adequately controlled, degrades the quality of life (QOL) of cancer patients. Although CINV is feared by cancer patients receiving highly-emetogenic chemotherapy (HEC), it can be controlled [2,3].

Based on phase III study data, the triple-drug combination of ondansetron (OND), dexamethasone (DEX), and aprepitant (APR) was regarded as the standard antiemetic regimen for cancer patients receiving HEC [4]. Palonosetron (PAL) has a longer half-life and stronger 5HT3 receptor binding affinity than other $5 \mathrm{HT} 3$ receptor antagonists (5HT3RAs), including OND, granisetron (GRA), and dolasetron (DOL) 
[5]. Moreover, PAL alone or in combination with DEX exhibited better antiemetic activity than GRA or DOL combined with DEX for the control of acute and delayed CINV in patients receiving moderately-emetogenic chemotherapy (MEC) $[6,7]$. National comprehensive cancer network guidelines currently recommend the combination of PAL, APR, and DEX (PAD) as a standard regimen for controlling HECinduced CINV in cancer patients [8]. However, there are no direct head-to-head comparisons of PAD vs. other 5HT3RAs.

Ramosetron (RAM), a 5HT3RA with a prolonged half-life and increased receptor binding affinity compared to OND or GRA [9], has been used widely for CINV prevention in Asia. The antiemetic activity of RAM was shown to be similar to that of PAL for controlling MEC-induced CINV [10].

The purpose of this phase IV study was to show the noninferiority of the combination of RAM, APR, and DEX (RAD) compared to PAD in controlling HEC-induced CINV.

\section{Materials and Methods}

\section{Study design}

This prospective, multicenter, single-blind, randomized phase IV clinical trial was conducted in 10 institutions. Patients were randomly assigned to RAD or PAD (1:1 ratio). The study was conducted under single-blind conditions, with participants not being aware of their assignment group.

Inclusion criteria included age 19-75 years, pathologicallyconfirmed malignant disease, and Eastern Cooperative Oncology Group performance status 0-2. Patients were scheduled to receive HEC on the first day of treatment. Patients were required to have adequate bone marrow, hepatic, and renal function. Major exclusion criteria included medications, medical illness, or medical conditions and procedures that could affect nausea or vomiting (a complete list of eligibility criteria is provided in the S1 Table).

\section{Study treatment}

APR (125 mg orally, 1 hour prior to chemotherapy, D1; 80 mg orally, D2-3) and DEX (12 mg, orally or intravenously, 30 minutes prior to chemotherapy, D1; $8 \mathrm{mg}$, orally, D2-4) were administered in both arms. The RAM and PAL arms received RAM (0.3 mg, D1) or PAL (0.25 mg, D1) intravenously 30 minutes before chemotherapy. Rescue antiemetics for severe nausea/vomiting were administered at the request of the patient or upon recommendation by the attending physicians at any time during the study period.

\section{Study endpoints}

The primary endpoint was overall complete response (CR), defined as no vomiting, including retching, and no requirement for rescue antiemetics within 5 days of HEC. Secondary endpoints were $\mathrm{CR}$, complete protection $(\mathrm{CP} ; \mathrm{CR}+$ nausea score $<25 \mathrm{~mm} ; 0-100 \mathrm{~mm}$ ), and total control (TC; CR+nausea score $<5 \mathrm{~mm} ; 0-100 \mathrm{~mm})$ in the acute (0-24 hours), delayed (D2-5), and overall (D0-5) periods; severity of nausea (determined using a $0-100 \mathrm{~mm}$ visual analog scale); time to first occurrence of vomiting; QOL assessed by the validated patient self-assessment Functional Living Index Emesis (FLIE) questionnaire [11]; safety. During the overall period, patients recorded daily episodes of vomiting or retching, the degree of nausea, and the use of rescue medication in a diary and using the Rhodes Index of Nausea and Vomiting Form2 (INV-2) [12]. Safety was evaluated based on clinical and laboratory adverse events (AEs) between the start day and the day before the next chemotherapy, assessed according to National Cancer Institute-Common Terminology Criteria for Adverse Events (NCI-CTCAE) ver. 4.03 [13].

\section{FLIE measurement and scoring}

FLIE includes 18 questions divided into two domains: nausea (questions 1-9) and vomiting (questions 10-18) [11]. The questionnaire was completed on D0 (reflecting daily-life activities before chemotherapy) and D6 (to provide information on the overall effect of CINV on daily-life activities during D1-5). The average score for each domain was summed and transformed using a pre-specified scoring procedure, which allowed for a minimum domain score of 6 and a maximum of 63. The chi-square test was used to compare the proportion of patients treated with RAM vs. PAL, with 'no impact on daily life' (NIDL) (i.e., individual question scores $>6$ on the 7-point FLIE scale, domain score $\geq 54$, overall FLIE score $\geq 108$ ) assessed for the FLIE domains of nausea, vomiting, and combined (i.e., total score).

\section{Statistical analysis}

The hypothesis was that the $\mathrm{CR}$ rate in the $\mathrm{RAD}$ arm during the overall period would not be inferior to the $C R$ rate in the PAD arm. A sample size of 135 in each arm allowed an $80 \%$ power and one-sided $\alpha$ level of 0.025 to detect a CR noninferiority difference of $-15 \%$ between the RAD and PAD groups, assuming the actual CR in each group to be $77 \%$ and $77 \%$, respectively. With an expected dropout rate of $15 \%$, sample size was increased to 146 patients per arm.

Stratified block randomization was conducted with a 1:1 ratio between groups, randomly mixing block sizes of 2 and 4 , considering (1) chemotherapeutic regimen (cisplatin vs. 


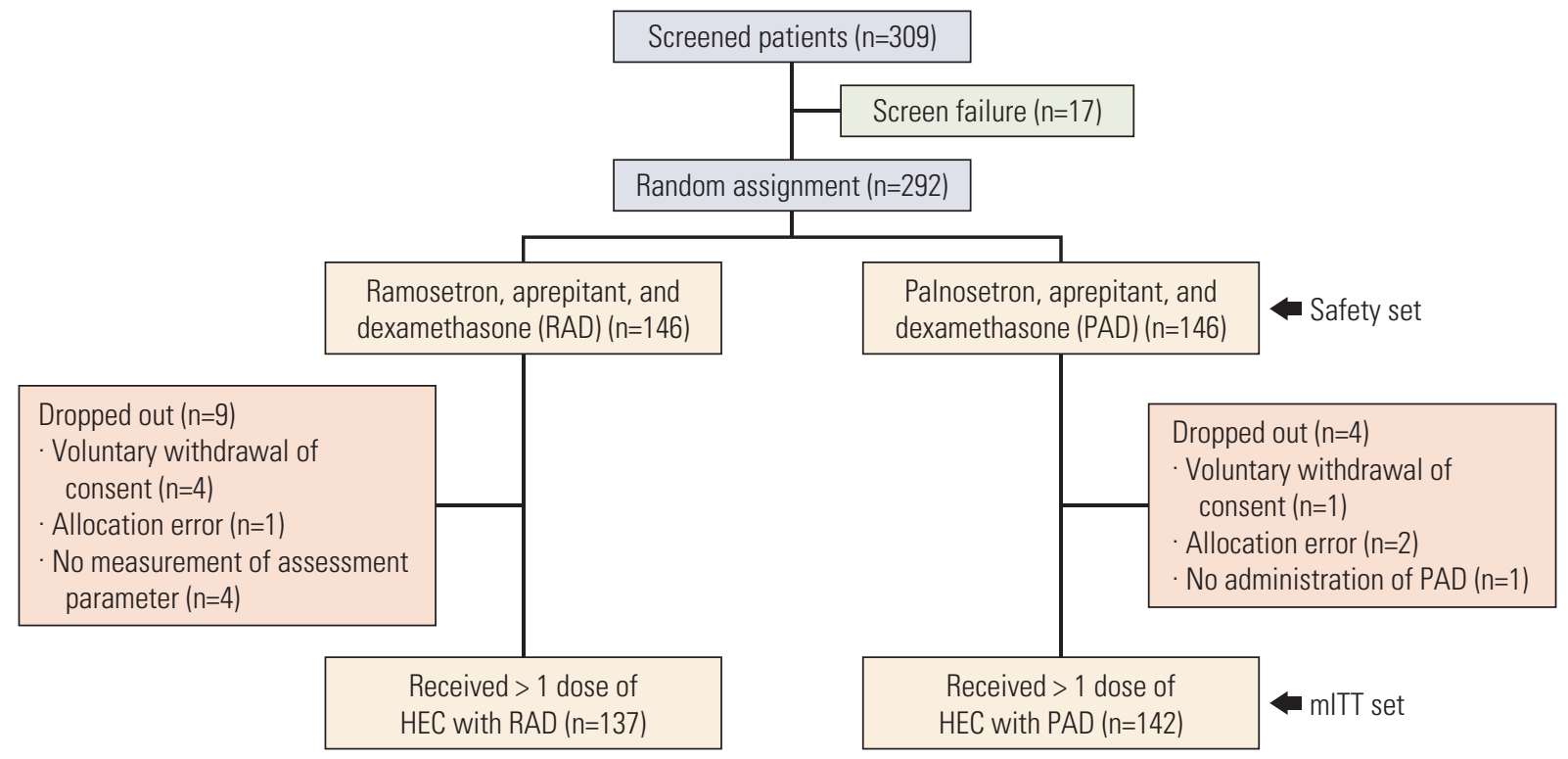

Fig. 1. Complete response rate (A), complete protection rate (B), and total control rate $(\mathrm{C})$ in intention-to-treat population $(\mathrm{n}=279)$ on a daily basis. (A) The risk difference between the two arms was $2.2 \%$ (95\% confidence interval [CI], $-7.1 \%$ to $11.4 \%$ ). (B) The risk difference between the two arms was $-2.3 \%$ (95\% CI, $-13.9 \%$ to $9.4 \%$ ). (C) The risk difference between the two arms was 3.8\% (95\% CI, $-7.9 \%$ to $15.5 \%$ ) using the generalized estimating equation model. HEC, highly-emetogenic chemotherapy; mITT, modified intention to treatment.

non-cisplatin), (2) treatment schedule (single-day vs. multiple-day), and (3) sex (male vs. female), as stratification factors. Patients were assigned according to a pre-defined randomization sequence created by an independent investigator with no clinical involvement in the trial.

Effectiveness was calculated in the modified intention-totreat population, defined as all patients randomly assigned to a treatment group having received at least one study treatment dose after randomization. Data were analyzed using SAS ver. 9.4 (SAS Institute Inc., Cary, NC). The two groups were compared using the chi-square test, Fisher exact test, and Wilcoxon rank-sum test, as appropriate.

\section{Ethical statement}

The study protocol was approved by the institutional review boards at the Seoul Saint Mary's Hospital (KC15MIMV0785), Kangdong Sacred Heart Hospital (KANGDONG2015-05-005), Kangbuk Samsung Hospital (KBSMC 2015-03010), Keimyung University Dongsan Hospital (DSMC 201506-060), St. Vincent's Hospital (VC16MIMV0059), Severance Hospital, Yonsei Cancer Center (4-2015-0414), Pusan National University Hospital (H-1506-003-044), Ajou University Hospital (AJIRB-MED-CT4-15-083), Chungnam National University Hospital (CNUH 2015-02-025), Samsung Medical
Center (SMC-2015-01-096) and registered with ClinicalTrials.gov (NCT02532634); all patients provided written informed consent.

\section{Results}

\section{Patients}

Between August 2015 and September 2017, a total of 309 patients were screened and 292 eligible patients were randomly assigned (1:1) to receive RAD or PAD (Fig. 1). Patient characteristics, disease demographics, chemotherapeutic regimens, and administration schedules were well balanced between both arms (Table 1).

\section{Efficacy}

The overall CR rates of RAD and PAD were $81.8 \%$ and $79.6 \%$ (risk difference [RD], 2.2\%; 95\% confidence interval [CI], -7.1 to 11.4). RAD was non-inferior to PAD, as evidenced by $\mathrm{CR}$ rates in the acute, delayed, and overall periods (RD, $2.0 \%, 4.4 \%$, and $2.2 \%$, respectively) (Table 2, Fig. 2A). 
Table 1. Demographics

\begin{tabular}{|c|c|c|c|}
\hline & RAD $(n=137)$ & $\operatorname{PAD}(n=142)$ & p-value \\
\hline \multicolumn{4}{|l|}{ Sex } \\
\hline Male & $86(62.8)$ & $87(61.3)$ & 0.796 \\
\hline Female & $51(37.2)$ & $55(38.7)$ & \\
\hline Age (yr) & $59.4 \pm 12.0$ & $60.3 \pm 11.8$ & 0.521 \\
\hline \multicolumn{4}{|l|}{ Motion sickness } \\
\hline Yes & $32(23.4)$ & $27(19.0)$ & 0.374 \\
\hline No & $105(76.6)$ & $115(81.0)$ & \\
\hline \multicolumn{4}{|l|}{ Alcohol drinking } \\
\hline Yes & $50(36.5)$ & $52(36.6)$ & 0.983 \\
\hline No & $87(63.5)$ & $90(63.4)$ & \\
\hline \multicolumn{4}{|l|}{ Tumor location } \\
\hline Lung and thymus & $59(43.1)$ & $61(42.9)$ & 0.074 \\
\hline Breast & $32(23.4)$ & $31(21.8)$ & \\
\hline Head and neck & $14(10.2)$ & $25(17.6)$ & \\
\hline GU and GY & $13(9.5)$ & $6(4.2)$ & \\
\hline Gastrointestinal & $9(6.6)$ & $3(2.1)$ & \\
\hline Other & $10(7.3)$ & $16(11.3)$ & \\
\hline \multicolumn{4}{|l|}{ Stage } \\
\hline I & $17(12.4)$ & $13(9.2)$ & 0.929 \\
\hline II & $28(20.4)$ & $32(22.5)$ & \\
\hline III & $36(26.3)$ & $37(26.1)$ & \\
\hline IV & $54(39.4)$ & $58(40.8)$ & \\
\hline NA & $2(1.5)$ & $2(1.4)$ & \\
\hline \multicolumn{4}{|c|}{ ECOG performance status } \\
\hline 0 & $51(37.2)$ & $51(35.9)$ & $0.466^{\mathrm{a})}$ \\
\hline 1 & $81(59.1)$ & $89(62.7)$ & \\
\hline 2 & $5(3.6)$ & $2(1.4)$ & \\
\hline \multicolumn{4}{|c|}{ Cisplatin-based chemotherapy $^{\text {b) }}$} \\
\hline Yes & $98(71.5)$ & $103(72.5)$ & 0.894 \\
\hline No & $39(28.5)$ & $39(27.5)$ & \\
\hline \multicolumn{4}{|c|}{ Administration schedule } \\
\hline Single day & $84(61.3)$ & $87(61.3)$ & $>0.99$ \\
\hline Multiple day & $53(38.7)$ & $55(38.7)$ & \\
\hline
\end{tabular}

Values are presented as number (\%) or mean \pm standard deviation. RAD, ramosetron, aprepitant, and dexamethasone; PAD, palonosetron, aprepitant, and dexamethasone; GU, genitourinary; GY, gynecology; Gastrointestinal, esophagus, stomach,

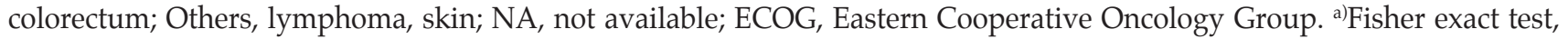
${ }^{b}$ Individual chemotherapy regimens are detailed in $\mathrm{S} 2 \mathrm{Table.}$

$\mathrm{CP}$ rates in RAD recipients were numerically lower than in PAD recipients, with efficacy differences in the acute, delayed and overall periods of $-1.5 \%,-2.8 \%$, and $-2.3 \%$, respectively (Table 2), but the non-inferiority of RAD vs. PAD remained unchanged (Fig. 2B).

TC rates in RAD during all study time periods were numerically higher than those in PAD, as evidenced by TC rates in the acute, delayed, and overall periods $(68.6 \%$ vs. $64.1 \%$ [RD $4.5 \%$ ], $48.9 \%$ vs. $46.5 \%$ [RD 2.4\%], and $47.5 \%$ vs. 43.7\% [RD 3.8\%], respectively) (Table 2, Fig. 2C).

\section{Quality of life}

The total FLIE score with RAD $(115.5 \pm 17.6)$ was comparable to that with PAD (114.8 $\pm 16.7, \mathrm{p}=0.745)$. The proportion of patients with FLIE score $\geq 108$ (NIDL) in RAD (75.0\%) was comparable to that in PAD (73.9\%) (RD, 1.09; 95\% CI, -9.32 to 11.49; $\mathrm{p}=0.838$ ). The proportion of patients with FLIE score $\geq 54$ in each of the nausea and vomiting domains in RAD was numerically higher than in PAD (72.2\% vs. $65.9 \%$ and $91.2 \%$ vs. $91.3 \%$, respectively), but not statistically significant 
Table 2. Complete response, complete protection, and total control rates in intention-to-treat population

\begin{tabular}{|c|c|c|c|}
\hline \multirow{2}{*}{ Phase } & \multicolumn{2}{|c|}{ No. $(\%)$} & \multirow{2}{*}{$\begin{array}{l}\text { Difference }^{\mathrm{a})} \\
(95 \% \mathrm{CI})\end{array}$} \\
\hline & RAD (n=137) & PAD $(n=142)$ & \\
\hline \multicolumn{4}{|l|}{ CR } \\
\hline Acute & 131 (95.6) & $133(93.7)$ & 2.0 (-3.3 to 7.2$)$ \\
\hline Delayed & $115(83.9)$ & $113(79.6)$ & $4.4(-4.7$ to 13.4$)$ \\
\hline Overall & $112(81.8)$ & $113(79.6)$ & $2.2(-7.1$ to 11.4$)$ \\
\hline \multicolumn{4}{|l|}{$\mathrm{CP}$} \\
\hline Acute & $105(76.6)$ & $111(78.2)$ & -1.5 (-11.3 to 8.3$)$ \\
\hline Delayed & $82(59.9)$ & $89(62.7)$ & $-2.8(-14.3$ to 8.6$)$ \\
\hline Overall & $77(56.2)$ & $83(58.5)$ & -2.3 (-13.9 to 9.4$)$ \\
\hline \multicolumn{4}{|l|}{$\mathrm{TC}$} \\
\hline Acute & $94(68.6)$ & $91(64.1)$ & 4.5 (-6.6 to 15.6$)$ \\
\hline Delayed & $67(48.9)$ & $66(46.5)$ & $2.4(-9.3$ to 14.2$)$ \\
\hline Overall & $65(47.5)$ & $62(43.7)$ & $3.8(-7.9$ to 15.5$)$ \\
\hline
\end{tabular}

RAD, ramosetron, aprepitant, and dexamethasone; PAD, palonosetron, aprepitant, and dexamethasone; 95\% CI, 95\% confidence interval; $\mathrm{CR}$, complete response; $\mathrm{CP}$, complete protection; $\mathrm{TC}$, total control. ${ }^{\mathrm{a}}$ Difference was calculated as (RAD-PAD).

( $\mathrm{p}=0.267$ and $\mathrm{p}=0.565$, respectively) (Table 3 ).

\section{Adverse events}

A total of 292 patients receiving RAD or PAD were evaluated in the safety cohort. Overall, no new safety signals were identified. Both RAD and PAD were well tolerated, with $>90 \%$ of AEs mild or moderate (Table 4 ). The most common hematologic and non-hematologic toxicities were neutropenia and anorexia, respectively.

\section{Discussion}

PAD is the standard regimen for controlling CINV in cancer patients receiving HEC. We conducted a prospective, multicenter, single-blind, randomized clinical study to prove the non-inferiority of RAD vs. PAD for the control of HECinduced CINV.

The distribution of patients between both treatment groups was well balanced by patient-related and chemotherapyrelated factors. The primary endpoint of the study was overall CR throughout 5 days post-HEC. The CR rate in PAD recipients, used as the reference arm, was comparable to rates in 1,453 patients who were randomized to PAD in seven randomized controlled trials (RCTs) for HEC-induced CINV [14-20]; CR rates in the RCTs vs. our study were $86.6 \%$ vs. $93.7 \%, 73.4 \%$ vs. $79.6 \%$, and $69.5 \%$ vs. $79.6 \%$ in the acute, delayed, and overall phases, respectively (Table 5). Mean- while, the overall CR rate (81.8\%) with RAD was slightly higher than in our previous phase III study $(77.1 \%)$ [21], but comparable to that with PAD (79.6\%). QOL with FLIE was very similar between RAD and PAD in terms of nausea, vomiting, and total score. In addition, the proportion of patients with FLIE score $\geq 108$ (NIDL) in RAD was comparable to that in PAD. These results strongly support the hypothesis that RAD is non-inferior to $\mathrm{PAD}$, as evidenced by FLIE score as well as CR rates in the acute, delayed, and overall phases.

$\mathrm{CR}, \mathrm{CP}$, and TC in RAD did not differ from those in PAD. The number of patients with a FLIE score in the nausea domain $\geq 54$ was also higher with RAD than PAD although it was not statistically significant. Considering that the definition of $\mathrm{CP}$ allows a low level of nausea, unlike TC, the mechanism of action of RAM may be different from PAL. Previously, Japanese researchers reported that RAM had higher affinity for cloned human and rat 5HT3 receptors, as well as extremely slower dissociation from the human $5 \mathrm{HT} 3$ receptor compared with other 5-HT3RAs including alosetron and cilansetron [22]. Accordingly, there are two possible explanations about the conflicting results in our study: (1) RAM may have stronger binding to $5 \mathrm{HT} 3$ receptors present on enterochromaffin cells; (2) RAM may block the extramembrane domain of $5 \mathrm{HT} 3$ receptor binding to serotonin more effectively.

Subgroup analyses by age, sex, chemotherapy regimen, and tumor type, proved that RAD is non-inferior to PAD. Although the subgroup analysis is controversial regarding statistical significance due to some results exceeding the noninferiority margin, RAD and PAD displayed distinct activi- 
A
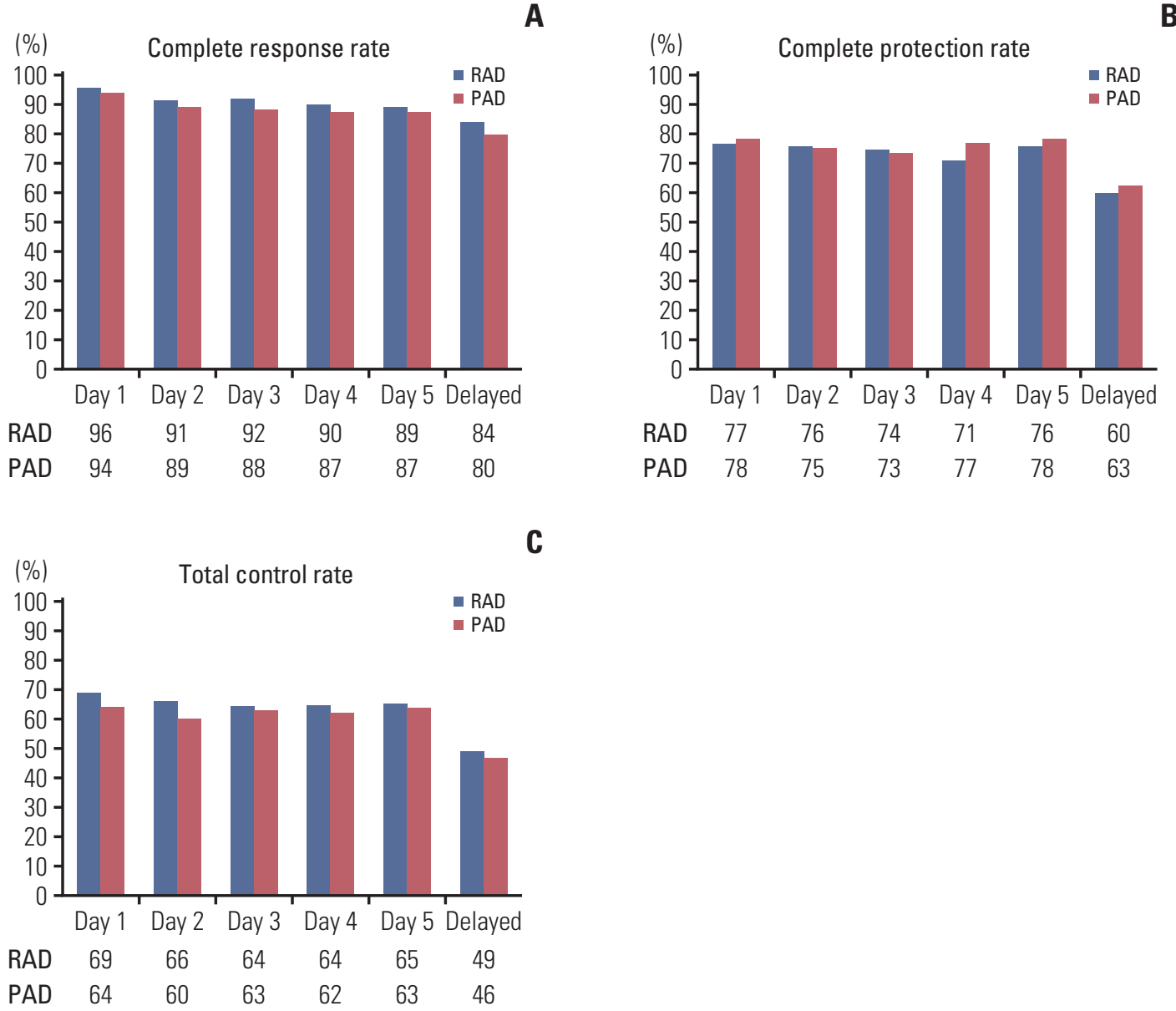

Fig. 2. Complete response rate (A), complete protection rate (B), and total control rate (C) in intention-to-treat population $(\mathrm{n}=279)$ on a daily basis. There was no statistical significance by individual date. (A) The risk difference between the two arms was 2.2\% (95\% confidence interval [CI], -7.1 to 11.4). (B) The risk difference between the two arms was $-2.3 \%$ (95\% $\mathrm{CI},-13.9$ to 9.4). (C) The risk difference between the two arms was $3.8 \%(95 \% \mathrm{CI},-7.9$ to 15.5$)$ using the generalized estimating equation model. RAD, ramosetron, aprepitant, and dexamethasone; PAD, palonosetron, aprepitant, and dexamethasone.

ties regarding different aspects of individual subgroups. Given the fact that cisplatin-containing HEC was administered for all lung cancer patients, RAM seems to be more effective for cisplatin-induced nausea and vomiting. Meanwhile, because non- cisplatin chemotherapy was given to all breast cancer patients, PAL seems to be more effective for non-cisplatin chemotherapy-induced nausea and vomiting.

FLIE scores in the total, nausea, and vomiting domains with RAD were quite similar to those with PAD. Patients with a FLIE total score $\geq 108$ (NIDL) and a FLIE score in the vomiting domain $\geq 54$ were comparable between both groups, but the number of patients with a FLIE score in the nausea domain $\geq 54$ was numerically higher in RAD than PAD. There is a possibility that the higher TC rate with RAD is reflected in a QOL benefit, with a correspondingly greater proportion of patients with NIDL due to more effective nausea control during the overall period. While this difference is small, it is encouraging that RAD has some potential to improve QOL.

In this study, no new safety signals were identified. More than $90 \%$ of AEs were mild or moderate in intensity. The incidence and duration of serious AEs or AEs was low and similar between both groups.

There are several study limitations. Firstly, because the study aim was to prove the non-inferiority of RAD vs. PAD, subgroup analysis was not scheduled. Although subgroup analysis produced some degree of differences between both arms, some analysis results did not show statistical significance due to the small number of subjects. Secondly, FLIE questionnaire results were not been collected on day 2 , 
Table 3. FLIE score and proportion of patients reporting NIDL for total, nausea domain, and vomiting domain in the mITT population

\begin{tabular}{|c|c|c|c|c|}
\hline & \multicolumn{2}{|c|}{ Mean \pm SD or $\mathrm{n}(\%)$} & \multirow{2}{*}{$\begin{array}{c}\text { Difference }^{\mathrm{a})} \\
(95 \% \mathrm{CI})\end{array}$} & \multirow{2}{*}{ p-value } \\
\hline & RAD (n=132) & PAD $(n=138)$ & & \\
\hline \multicolumn{5}{|l|}{ FLIE score } \\
\hline Total & $115.5 \pm 17.6$ & $114.8 \pm 16.7$ & $0.68(-3.43$ to 4.79$)$ & 0.745 \\
\hline Nausea domain & $54.3 \pm 13.9$ & $53.8 \pm 13.0$ & $0.48(-2.74$ to 3.70$)$ & 0.770 \\
\hline Vomiting domain & $61.2 \pm 6.5$ & $61.0 \pm 6.8$ & $0.27(-1.33$ to 1.87$)$ & 0.742 \\
\hline \multicolumn{5}{|l|}{ NIDL } \\
\hline Total $\geq 108$ & $99(75.0)$ & $102(73.9)$ & $1.09(-9.32$ to 11.49$)$ & 0.838 \\
\hline Nausea domain $\geq 54$ & $95(72.0)$ & $91(65.9)$ & $6.24(-4.74$ to 17.22$)$ & 0.267 \\
\hline Vomiting domain $\geq 54$ & $123(93.2)$ & $126(91.3)$ & $1.88(-4.49$ to 8.25$)$ & 0.565 \\
\hline
\end{tabular}

FLIE score, the Functional Living Index-Emesis score; NIDL, no impact on daily life; mITT, modified intention to treatment; $\mathrm{SD}$, standard deviation; RAD, ramosetron, aprepitant, and dexamethasone; PAD, palonosetron, aprepitant, and dexamethasone; $\mathrm{CI}$, confidence interval. ${ }^{\mathrm{a}}$ Difference was calculated as (RAD-PAD).

Table 4. Safety profile (any adverse events with incidence $>1 \%$ )

\begin{tabular}{lccc} 
& RAD (n=146) & PAD (n=145) & p-value \\
\hline Leukopenia & $35(24.0)$ & $38(26.1)$ & $>0.05$ \\
Neutropenia & $41(28.1)$ & $46(31.6)$ & $>0.05$ \\
Febrile neutropenia & $12(8.3)$ & $9(6.2)$ & $>0.05$ \\
\hline Anemia & $30(20.6)$ & $29(19.9)$ & $>0.05$ \\
\hline Thrombocytopenia & $21(14.4)$ & $17(11.7)$ & $>0.05$ \\
\hline Anorexia & $43(29.5)$ & $47(32.2)$ & $>0.05$ \\
Mucositis & $14(9.6)$ & $19(13.1)$ & $>0.05$ \\
\hline Diarrhea & $18(12.4)$ & $13(9.0)$ & $>0.05$ \\
\hline Abdominal pain & $9(6.2)$ & $7(4.8)$ & $>0.05$ \\
\hline Constipation & $24(16.5)$ & $26(17.9)$ & $>0.05$ \\
\hline Alopecia & $27(18.5)$ & $40(27.4)$ & $>0.05$ \\
\hline Rash & $4(2.8)$ & $4(2.8)$ & $>0.05$ \\
\hline AST elevation & $5(3.5)$ & $5(3.5)$ & $>0.05$ \\
\hline ALT elevation & $6(4.2)$ & $4(2.8)$ & $>0.05$ \\
\hline Hypernatremia & $8(5.5)$ & $11(7.6)$ & $>0.05$ \\
\hline Hyperkalemia & $12(8.3)$ & $13(9.0)$ & $>0.05$ \\
\hline Hypercalcemia & $8(5.5)$ & $8(5.5)$ & $>0.05$ \\
\hline Hypophosphatemia & $9(6.2)$ & $5(3.5)$ & $>0.05$ \\
\hline
\end{tabular}

Values are presented as number (\%). Adverse events were graded according to National Cancer Institute-Common Terminology Criteria (CTCAE) ver. 4.03. RAD, ramosetron, aprepitant, and dexamethasone; PAD, palonosetron, aprepitant, and dexamethasone; AST, aspartate aminotransferase; ALT, alanine aminotransferase.

reflecting QOL during the acute period. Delayed emesis negatively influences patient QOL during administration of HEC and beyond. Both 5HT3RAs demonstrated significant inhibition against HEC-induced CINV, but did not improve control of delayed nausea, indicating that unmet needs remain for novel antiemetics.
In conclusion, in all aspects of efficacy, safety, and QOL, our data suggested that RAD was comparable to PAD for controlling HEC-CINV in cancer patients. 


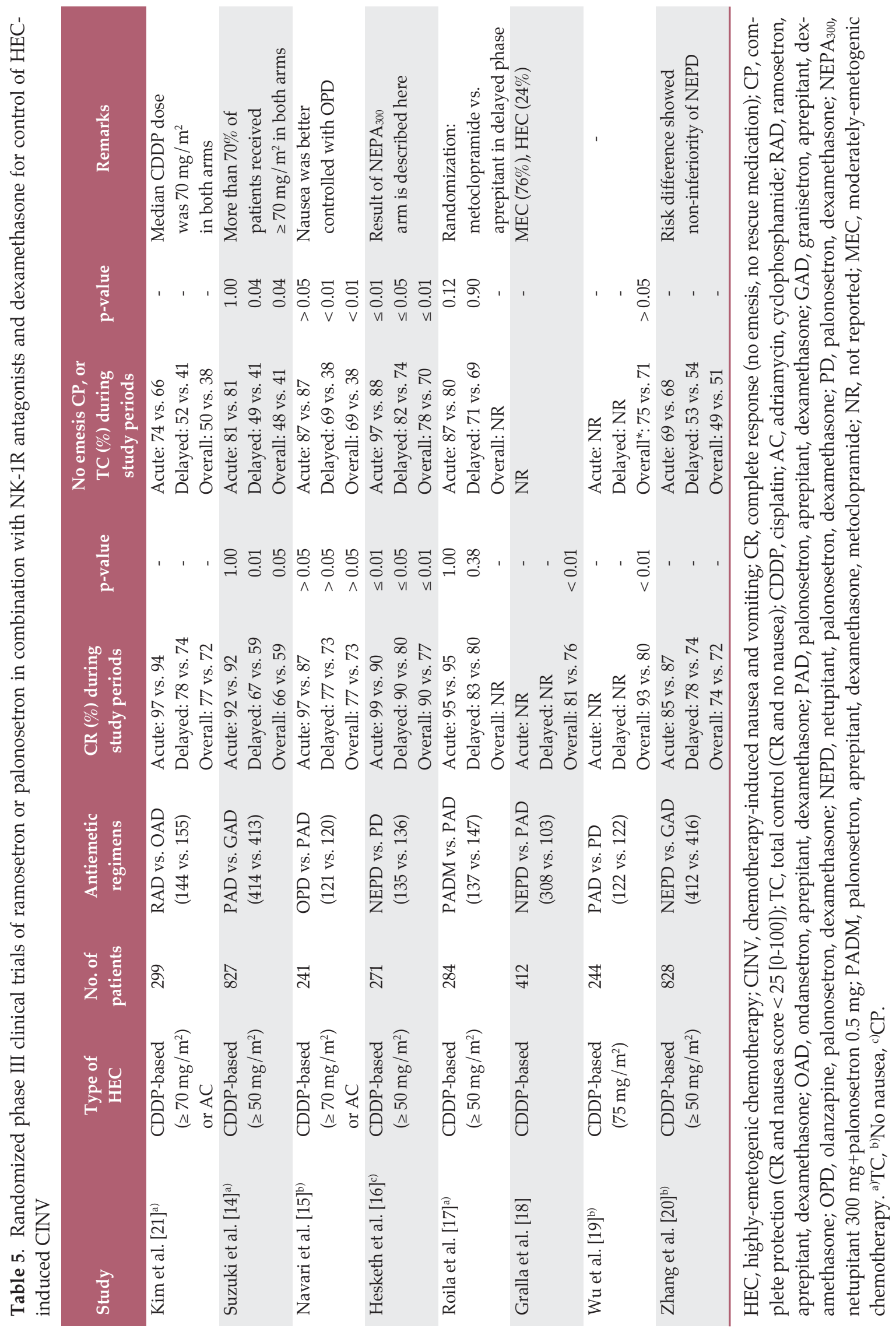




\section{Electronic Supplementary Material}

Supplementary materials are available at Cancer Research and Treatment website (https://www.e-crt.org).

\section{Conflicts of Interest}

JH Kang has acted as an advisor for Amgen, Roche, Merck, MSD, Ono/BMS, AstraZeneca, YooHan, SL Bigen, has received research funding from AstraZeneca, Boehringer Ingelheim, Ono, Yoohan, and ChongKunDang, and has acted as a speaker for AstraZeneca, Roche, Merck, and Boehringer Ingelheim. JH Sohn has received research funding from MSD, Roche, Novartis, AstraZeneca, Lilly, Pfizer, Bayer, GSK, CONTESSA, and Daiichi Sankyo. JS Ahn reports personal fees from Amgen, personal fees from Pfizer, personal fees from AstraZeneca, personal fees from Menarini, personal fees from Roche, personal fees from Eisai, personal fees from Boehringer Ingelheim, personal fees from BMS-Ono, personal fees from MSD, personal fees from Janssen, personal fees from Samsung Bioepis, outside the submitted work. All remaining authors declare no conflicts of interest.

\section{Acknowledgments}

The authors thank the clinical investigators, patients, and their families. They also acknowledge the hard work and great effort of the site clinical research nurses who participated in the study: So Hee Park, Hee Yeon Nam, Ji Yun Won, Su Jung Han, Haejeung Kim, Yujeoung Park, Ehyun Choi, Miseon Yu, Da Yeon Gwak, So Ra Jeong, Yang Soon Kim, Hwa Young Lee, Seryun Kang, Eun Hee Cho, Eun Joo Hong, Jin Joo Hong. Editorial support was provided, under the guidance of the authors, by David P. Figgitt PhD, ISMPP CMPPтм, Content Ed Net, with funding from Astellas Pharma Korea, Inc.

\section{Author Details}

${ }^{1}$ Division of Medical Oncology, Department of Internal Medicine, Seoul St. Mary's Hospital, College of Medicine, The Catholic University of Korea, Seoul, ${ }^{2}$ Division of Hemato-Oncology, Department of Internal Medicine, Kangdong Sacred Heart Hospital, Hallym University College of Medicine, Seoul, ${ }^{3}$ Division of Hematology / Oncology, Department of Internal Medicine, Kangbuk Samsung Hospital, Sungkyunkwan University School of Medicine, Seoul, ${ }^{4}$ Division of Hematology / Oncology, Department of Internal Medicine, Keimyung University Dongsan Hospital, Keimyung University School of Medicine, Daegu, ${ }^{5}$ Division of Medical Oncology, Department of Internal Medicine, St. Vincent's Hospital, College of Medicine, The Catholic University of Korea, Seoul, ' Division of Medical Oncology, Department of Internal Medicine, Yonsei University College of Medicine, Seoul, ${ }^{7}$ Division of Hemato-Oncology, Department of Internal Medicine, Pusan National University Hospital, Pusan National University School of Medicine, Busan, ${ }^{8}$ Department of Hematology-Oncology, Ajou University Hospital, Ajou University School of Medicine, Suwon, ${ }^{9}$ Department of Internal Medicine, Chungnam National University Hospital, Chungnam National University School of Medicine, Daejeon, ${ }^{10}$ Division of Hematology and Oncology, Department of Medicine, Samsung Medical Center, Sungkyunkwan University School of Medicine, Seoul, ${ }^{11}$ Department of Clinical Epidemiology and Biostatistics, Asan Medical Center, University of Ulsan College of Medicine, Seoul, Korea

\section{References}

1. Lindley C, McCune JS, Thomason TE, Lauder D, Sauls A, Adkins S, et al. Perception of chemotherapy side effects cancer versus noncancer patients. Cancer Pract. 1999;7:59-65.

2. Passik SD, Kirsh KL, Rosenfeld B, McDonald MV, Theobald DE. The changeable nature of patients' fears regarding chemotherapy: implications for palliative care. J Pain Symptom Manage. 2001;21:113-20.

3. Fernandez-Ortega P, Caloto MT, Chirveches E, Marquilles R, Francisco JS, Quesada A, et al. Chemotherapy-induced nausea and vomiting in clinical practice: impact on patients' quality of life. Support Care Cancer. 2012;20:3141-8.

4. Hesketh PJ, Grunberg SM, Gralla RJ, Warr DG, Roila F, de Wit $\mathrm{R}$, et al. The oral neurokinin- 1 antagonist aprepitant for the prevention of chemotherapy-induced nausea and vomiting: a multinational, randomized, double-blind, placebo-controlled trial in patients receiving high-dose cisplatin--the Aprepitant
Protocol 052 Study Group. J Clin Oncol. 2003;21:4112-9.

5. Grunberg SM, Koeller JM. Palonosetron: a unique 5-HT3receptor antagonist for the prevention of chemotherapyinduced emesis. Expert Opin Pharmacother. 2003;4:2297-303.

6. Eisenberg P, Figueroa-Vadillo J, Zamora R, Charu V, Hajdenberg J, Cartmell A, et al. Improved prevention of moderately emetogenic chemotherapy-induced nausea and vomiting with palonosetron, a pharmacologically novel 5 -HT3 receptor antagonist: results of a phase III, single-dose trial versus dolasetron. Cancer. 2003;98:2473-82.

7. Gralla R, Lichinitser M, Van Der Vegt S, Sleeboom H, Mezger J, Peschel C, et al. Palonosetron improves prevention of chemotherapy-induced nausea and vomiting following moderately emetogenic chemotherapy: results of a double-blind randomized phase III trial comparing single doses of palonosetron with ondansetron. Ann Oncol. 2003;14:1570-7. 
8. National Comprehensive Cancer Network. Antiemesis (version 1.2020) [Internet]. Plymouth Meeting, PA: National Comprehensive Cancer Network; 2020 [cited 2020 Feb 27]. Available from: http://www.nccn.org/professionals/ physician_gls/pdf/antiemesis.pdf.

9. Rabasseda X. Ramosetron, a 5-HT3 receptor antagonist for the control of nausea and vomiting. Drugs Today (Barc). 2002;38: 75-89.

10. Kim JS, Kim JY, Lee SJ, Park DK, Namgung H, Kim CN, et al. Multicenter nonrandomized trial of ramosetron versus palonosetron in controlling chemotherapy-induced nausea and vomiting for colorectal cancer. Ann Surg Treat Res. 2014;87:913.

11. Martin AR, Pearson JD, Cai B, Elmer M, Horgan K, Lindley C. Assessing the impact of chemotherapy-induced nausea and vomiting on patients' daily lives: a modified version of the Functional Living Index-Emesis (FLIE) with 5-day recall. Support Care Cancer. 2003;11:522-7.

12. Rhodes VA. Criteria for assessment of nausea, vomiting, and retching. Oncol Nurs Forum. 1997;24(7 Suppl):13-9.

13. National Cancer Institute-Common Terminology Criteria for Adverse Events (NCI-CTCAE) [Internet]. Bethesda, MD: National Cancer Institute; 2010 [cited 2019 Sep 11]. Available from: http://evs.nci.nih.gov/ftp1/CTCAE/CTCAE_4.03_201006-14_QuickReference_5x7.pdf.

14. Suzuki K, Yamanaka T, Hashimoto H, Shimada Y, Arata K, Matsui R, et al. Randomized, double-blind, phase III trial of palonosetron versus granisetron in the triplet regimen for preventing chemotherapy-induced nausea and vomiting after highly emetogenic chemotherapy: TRIPLE study. Ann Oncol. 2016;27:1601-6.

15. Navari RM, Gray SE, Kerr AC. Olanzapine versus aprepitant for the prevention of chemotherapy-induced nausea and vomiting: a randomized phase III trial. J Support Oncol. 2011;9:18895.

16. Hesketh PJ, Rossi G, Rizzi G, Palmas M, Alyasova A, Bondarenko I, et al. Efficacy and safety of NEPA, an oral combi- nation of netupitant and palonosetron, for prevention of chemotherapy-induced nausea and vomiting following highly emetogenic chemotherapy: a randomized dose-ranging pivotal study. Ann Oncol. 2014;25:1340-6.

17. Roila F, Ruggeri B, Ballatori E, Fatigoni S, Caserta C, Licitra L, et al. Aprepitant versus metoclopramide, both combined with dexamethasone, for the prevention of cisplatin-induced delayed emesis: a randomized, double-blind study. Ann Oncol. 2015;26:1248-53.

18. Gralla RJ, Bosnjak SM, Hontsa A, Balser C, Rizzi G, Rossi G, et al. A phase III study evaluating the safety and efficacy of NEPA, a fixed-dose combination of netupitant and palonosetron, for prevention of chemotherapy-induced nausea and vomiting over repeated cycles of chemotherapy. Ann Oncol. 2014;25:1333-9.

19. Wu F, Lin X, Yang Z, Sun Z, Zeng F, Heng J, et al. Phase III randomized trial of palonosetron and dexamethasone with or without aprepitant to prevent nausea and vomiting induced by full-dose single-day cisplatin-based chemotherapy in lung cancer. Clin Lung Cancer. 2018;19:e913-8.

20. Zhang L, Lu S, Feng J, Dechaphunkul A, Chang J, Wang D, et al. A randomized phase III study evaluating the efficacy of single-dose NEPA, a fixed antiemetic combination of netupitant and palonosetron, versus an aprepitant regimen for prevention of chemotherapy-induced nausea and vomiting (CINV) in patients receiving highly emetogenic chemotherapy (HEC). Ann Oncol. 2018;29:452-8.

21. Kim HJ, Shin SW, Song EK, Lee NR, Kim JS, Ahn JS, et al. Ramosetron versus ondansetron in combination with aprepitant and dexamethasone for the prevention of highly emetogenic chemotherapy-induced nausea and vomiting: a multicenter, randomized phase III trial, KCSG PC10-21. Oncologist. 2015;20:1440-7.

22. Hirata T, Keto Y, Funatsu T, Akuzawa S, Sasamata M. Evaluation of the pharmacological profile of ramosetron, a novel therapeutic agent for irritable bowel syndrome. J Pharmacol Sci. 2007;104:263-73. 